Musées, Patrimoine et Culture scientifiques et techniques

$158 \mid 2015$

mars-avril 2015

\title{
Plus de bruits au musée
}

Marc Touché

URL : http://journals.openedition.org/ocim/1489

DOI : $10.4000 /$ ocim. 1489

ISSN : 2108-646X

Éditeur

OCIM

Édition imprimée

Date de publication : 1 mars 2015

Pagination : 5-12

ISSN : 0994-1908

Référence électronique

Marc Touché, "Plus de bruits au musée », La Lettre de I'OCIM [En ligne], 158 | 2015, mis en ligne le 01 mars 2016, consulté le 01 mai 2019. URL : http://journals.openedition.org/ocim/1489 ; DOI : 10.4000/ ocim. 1489

Ce document a été généré automatiquement le 1 mai 2019.

Tous droits réservés 


\title{
Plus de bruits au musée
}

\author{
Marc Touché
}

Présentation au Mupop de guitares électriques « artisanales » utilisées lors de pratiques musicales amateurs.

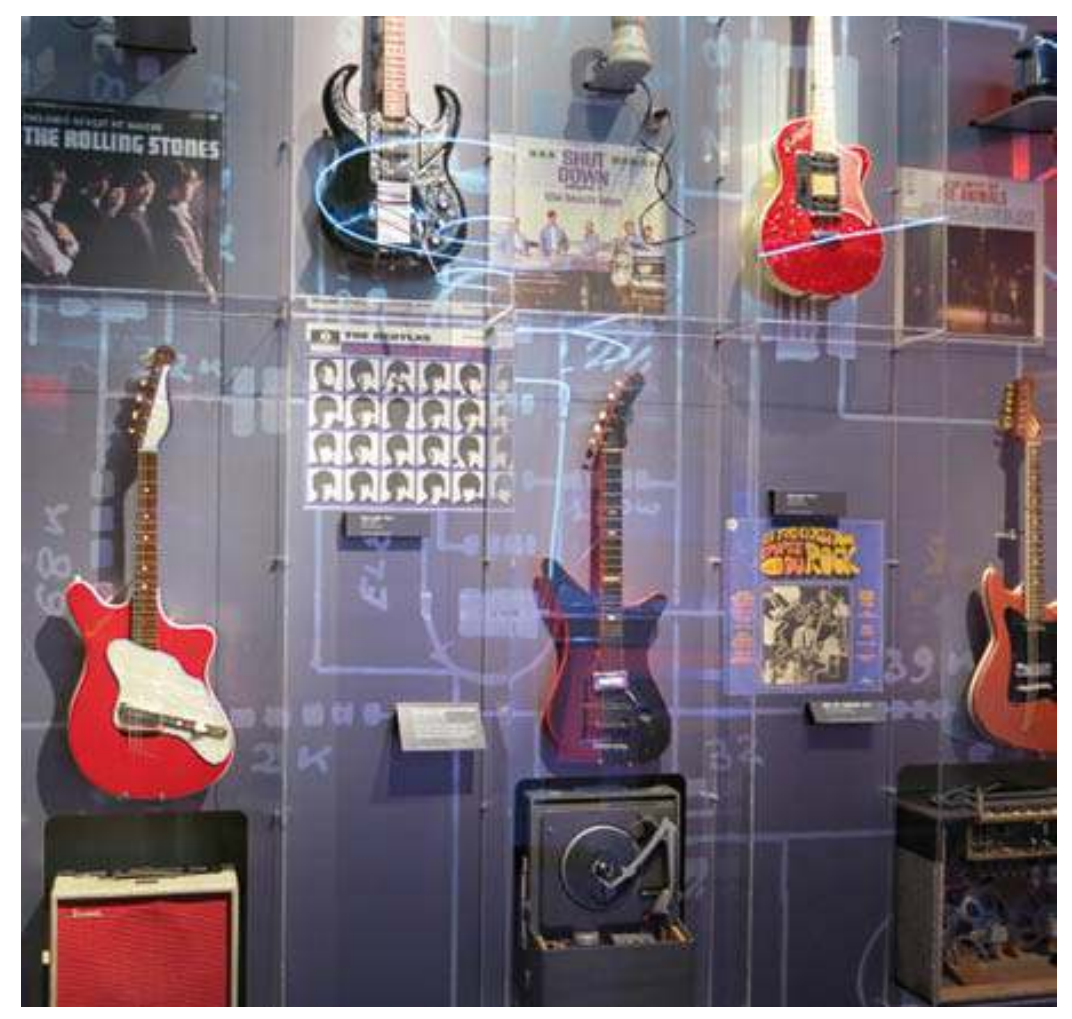

(c) $\mathrm{OCIM} / \mathrm{SL}$ 


\section{Quelle place dans les musées pour la musicalisation électroamplifiée des façons de vivre?}

1 Les musiques populaires et leur mode de socialisation sont, dans la seconde partie du XXe siècle, le théâtre de grands bouleversements. Le phénomène sociologique des groupes de guitares électriques autodidactes et auto proclamés musiciens est dès le début des années 1960 une première grande manifestation de l'entrée de la jeunesse comme acteur autonome dans ce paysage musical. En quelques décennies «un processus de musicalisation électroamplifiée des mœurs » va parcourir et pénétrer tous les milieux de la société française et tout particulièrement les espaces domestiques pour l'écoute, la pratique instrumentale et la répétition en groupe. La question des niveaux sonores - la musique des uns devenant du bruit pour les autres - amène les groupes à faire feu de tout bois pour trouver un lieu pour jouer de la musique. Dans cet article, à partir d'une aventure originale, celle du groupe dit "des Crocos», je vais aborder la question suivante : « Fête de la musique, faites toutes les musiques » tel que le propose des affiches institutionnelles de la Fête de la musique du 21 juin chaque année, oui mais où pour les créer et s'entraîner? Comment les musiciens électroamplifiés trouvent-ils des solutions en milieu urbain? Les jeunes rockers d'hier vieillissent souvent avec leur passion du temps de l'adolescence. Ces pratiques concernent aujourd'hui toutes les classes d'âges, de l'adolescence aux retraités et tous les milieux sociaux, mais principalement le sexe masculin. Quelle place accorder, dans les lieux de mémoire que sont les musées, à ces pratiques, ces objets, ces cultures matérielles, ces sociabilités, ces petites productions musicales artisanales? Comment les exposer sans trop les trahir dans les expositions temporaires et les parcours permanents ? Voici donc un bout de l'histoire de l'entrée des problématiques d'une "unité écologique » électroamplifiée dans les collections et parcours permanents du Mupop de Montluçon.

2 Après des années d'enquêtes de terrains et d'expositions sur ces domaines, j'avais acquis l'intime conviction, et je l'avais partagée avec l'équipe et les deux directeurs du Mupop (Sylvie Douce de la Salle et Éric Bourgougnon), qu'il fallait ne pas isoler des objets qui dans la vraie vie sont en interactions et communiquent entre eux, se répondent, fusionnent leurs sonorités. Nous retenions l'idée de les présenter dans le contexte dans lequel ils sont utilisés, dans «leur jus ». Le temps et le lieu de répétition constituent le dénominateur commun à toutes ces pratiques collectives depuis plus de cinq décennies, en effet tous les groupes n'ont pas pour finalité de jouer en concert et d'enregistrer en studio, mais tous répètent et créent collectivement en répétition. J'avais donc construit d'une part ce temps social méconnu en temps fort des pratiques et d'autre part, le lieu dit de « répète » en haut-lieu des cuisines et coulisses des musiques amplifiées, là où cultures matérielles et immatérielles se fondent pour donner naissance à des esthétiques musicales dont les slogans de certaines sont "plus de bruit ». Les Crocos appartiennent à ces réseaux musicaux. Ceux du rock alternatif français et international, contestataire et antiraciste. Quand le local est refermé à clef sur le groupe, il devient un lieu d'intimité et de liberté, il est le creuset du processus de création collective.

3 Les musiques - pour dire vite et par commodité, dites « rock » - vont passer en France des marges, voire de l'illégitimité culturelle au stade de la culture de masse et de la reconnaissance institutionnelle encore balbutiante du côté des pouvoirs publics. Dans cet article, à partir de l'exemple de la recherche collecte et de la patrimonialisation puis de 
l'exposition au public d'un local punk-rock dit couramment «des Crocos ", il s'agira de montrer à la fois quels sont les principes et les enjeux d'une activité muséale orientée vers des objets aussi peu habituels que les musiques électroamplifiées ${ }^{2}$ : plus largement, comment peut s'élaborer une anthropologie des univers sonores du rock, qui renseigne sur les sociabilités (principalement mais pas exclusivement de la jeunesse), sur les modes de vie (la question des loisirs, du défoulement, du divertissement, la culture du potentiomètre), sur le rapport aux espaces publics et privés (les usages sonores, vibratoires qui transgressent l'ordre des murs), sur les rapports au corps (tatouages en lien avec la musique, pratiques sonores paroxystiques, cultures décibéliques et vibrationistes ${ }^{3}$, stylisation des apparences), sur la définition des domaines artistiques (du punk au rock virtuose, savant), sur les aspects économiques (autoproduction/artisanat, industries du spectacle, des instruments et du disque), et enfin sur les rapports sociaux (la contestation, l'auto organisation, les circuits alternatifs). La musique est souvent pensée en oubliant les musiciens en chair et en os, chargés de culture, en oubliant ou en laissant dédaigneusement de côté les outils, les lieux, contextes sociaux et physiques. Le travail avec les musées est l'occasion de remettre de façon sensible au centre du débat les musiciens, leurs musiques, leurs outils et leurs publics.

Dans le local d'origine, aux Mureaux (78) l'un des membres fondateurs des Crocos

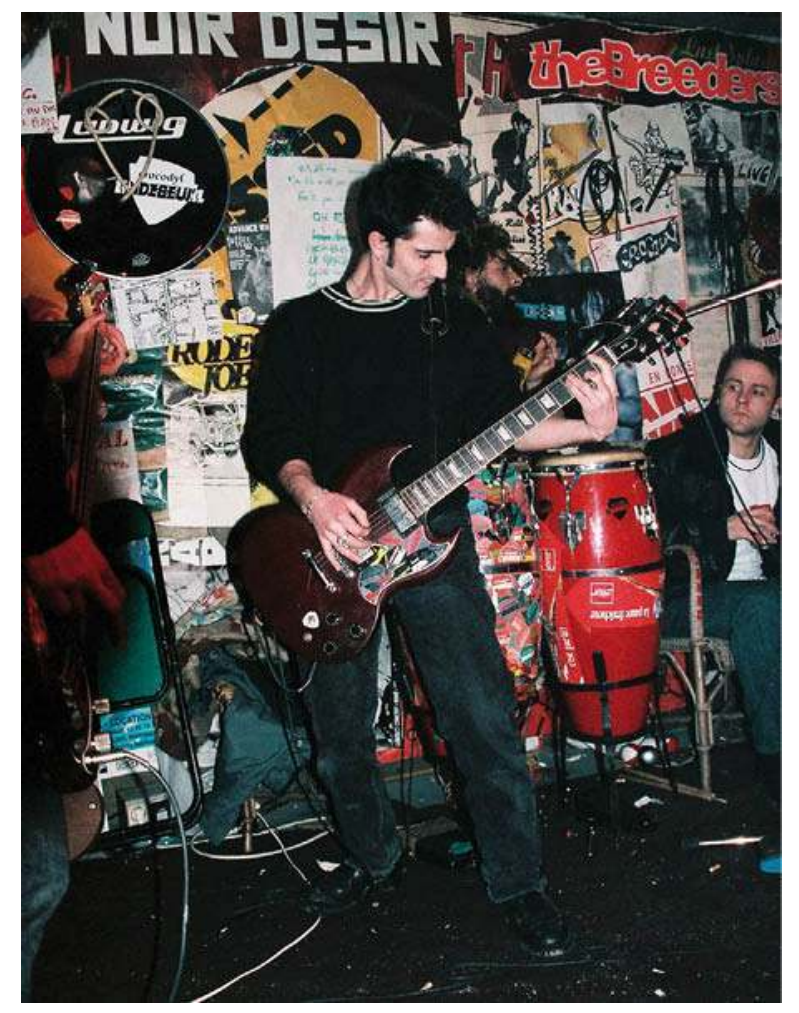

(c) Marc Touché, CNRS/Mupop

\section{Muséographier des « musiques électroamplifiées » dans le cadre de recherche-collecte}

Cet article essaie aussi de rendre compte d'une aventure intellectuelle particulière : la création de collections d'objets, d'archives écrites et sonores concernant les mondes et 
l'histoire des «musiques électroamplifiées " à partir de recherches sociologiques de terrain inscrites dans la durée en France. Elles ont été créées en tenant compte de l'état des collections du musée de la Musique de Paris, dans un contexte de travail associant deux laboratoires du CNRS (le Centre d'ethnologie française et le laboratoire Georges Friedmann dans lesquels je travaillais), et trois musées: deux musées municipaux (le musée des Musiques populaires de Montluçon dans l'Allier qui devient en 2013 le Mupop, le musée château d'Annecy en Haute-Savoie ${ }^{4}$ ) et un musée national des Arts et Traditions populaires à Paris qui devient en 2013 le MuCEM à Marseille.

5 Les travaux ${ }^{5}$ que j'ai menés sur la place de la musique dans la vie de jeunes dans des ensembles HLM de la banlieue parisienne m'ont conduit progressivement à m'intéresser, dans les années 1980, aux conditions de pratiques collectives de la musique en dehors des contextes institutionnels: des recherches pour le ministère de l'Environnement dans les années 1990 sur les questions de la connaissance de l'environnement sonore urbain à partir des lieux de répétition amplifié et de la gestion des risques auditifs par les musiciens de musiques amplifiées, ainsi que des recherches et expositions sur l'histoire locale : en région parisienne, à Agen, Annecy ${ }^{6}$, Laval ${ }^{7}$, Limoges, Montluçon, Tulle ${ }^{8}$. En 1992 j'avais écrit un très court article dont je ne pouvais imaginer qu'il préfigurait ma vie professionnelle des années à venir («Une histoire à construire la mémoire des musiques amplifiées hier et demain ») à l'occasion de l'inauguration du Florida à Agen, première politique publique dédiée en France aux «musiques amplifiées». À cette époque je commençais des enquêtes sur les groupes de musiques amplifiées de Montluçon. Ma vie de sociologue allait totalement basculer. Le musée des Musiques populaires de Montluçon possédait de très belles et importantes collections de cornemuses et vielles à roue. Sa conservatrice Sylvie Douce de la Salle et l'adjoint à la culture François Laplanche souhaitaient étendre les collections à la période contemporaine et pourquoi pas au rock. Un travail d'équipe sur les musiques amplifiées avec l'ensemble des personnels de ce musée s'est alors mis en place et se poursuit depuis quinze années avec le nouveau conservateur Éric Bourgougnon et l'adjointe à la culture de l'Agglomération Évelyne Tautou. Dès 1994, ce travail a mené à la création des premières grandes collections de «musiques amplifiées» en France, documentées par des recherches in situ et inscrites dans la durée en divers territoires du pays : la première étape fut l'acquisition audacieuse pour l'époque d'une " unité écologique » : un local de répétition de sociabilité punk-rock d'adulte, de sexe masculin, celui du groupe Crocodyl Lindebeulll; suivi de celle de dix mille cassettes et cd produites artisanalement par des groupes amateurs, puis de celle d'un studio d'enregistrement associatif parisien, le studio Campus ; d'un instrumentarium électrique de musiciens de rue en situation sociale précaire, le groupe rock du sud-est Kalimutcho ; de deux instrumentariums de groupes pionniers du rock montluçonnais, Les Rand'gers pour le début des années 1960 et Les Bretzel Liquide pour les années 1970; ainsi que des séries d'instruments et des archives de fabricants concernant la lutherie électrique française des années 1940 à 1980 : Stimer, RV, Garen, MI, Jacobacci ${ }^{9}$, Benedetti/ Golden-Sound. Ce travail a été étendu au domaine de la batterie avec les marques Asba, Cirichelli-Capelle dont l'histoire traverse une grande partie du XXe siècle. 


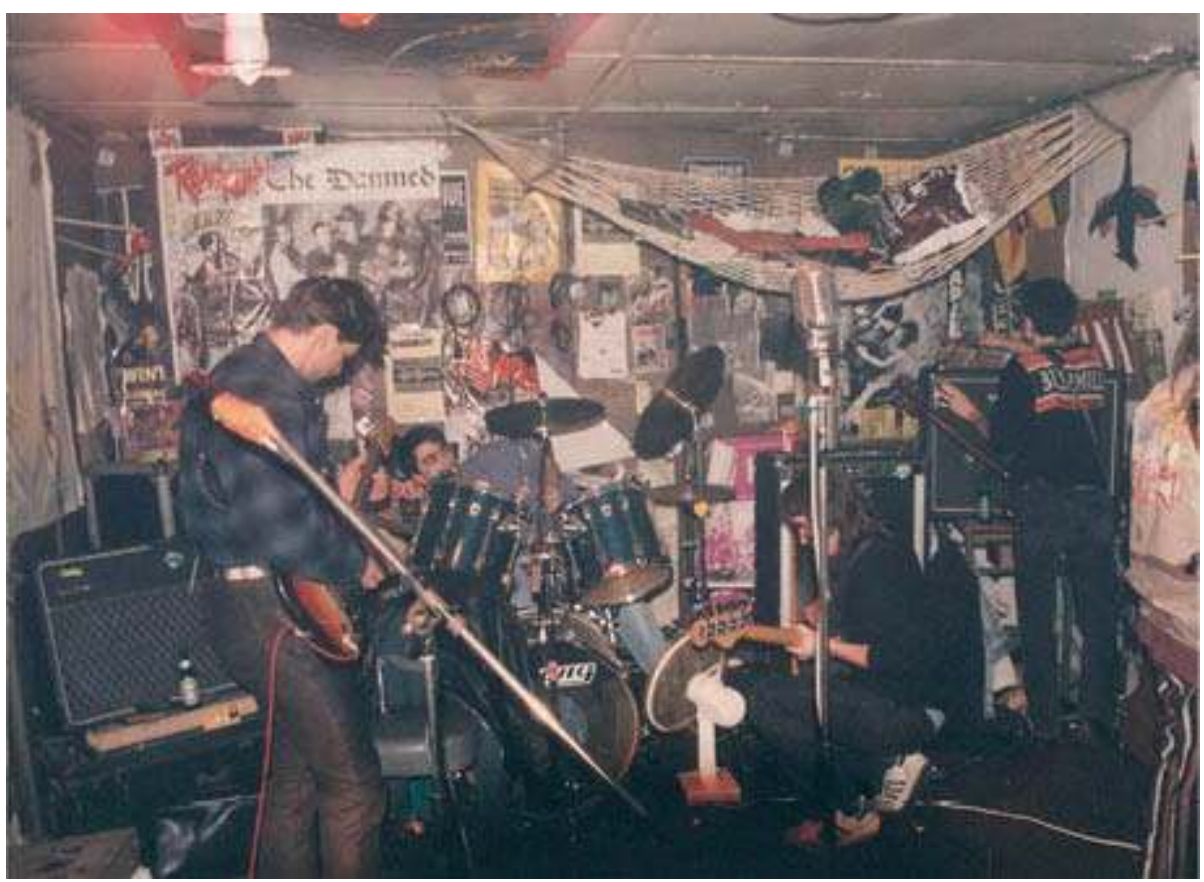

(C) Marc Touché, CNRS/Mupop

\section{Un local de répétition?}

Suivant les principes $d u$ «do it yourself» punk, ce local témoigne d'une façon personnalisée et intime de créer un lieu pour y faire librement de la musique sans déranger le voisinage. Les musiciens de ce groupe sont issus de milieux sociaux modestes, humbles. Au moment de l'acquisition de ce local de répétition par le musée, le groupe était composé de Poulos au chant, Will, Pat et Ludo aux guitares électriques, Gino à la batterie, Ras Jean-Phi aux percussions, Riton à la basse électrique et Rodolphe au violon. Le groupe est fondé à l'occasion d'une Fête de la musique un 21 juin au cœur des années 1980 pour un concert improvisé dans un bar. Il réunit des musiciens aux parcours très diversifiés : autodidactes, fanfare et conservatoire de musique. Le groupe se revendique de la famille punk-rock, mais l'ensemble de leurs influences représente une cuisine musicale assez complexe qui mêle les parfums sonores et les rythmes du blues, du rock'n'roll, du rock, du hard-rock, du reggae, du punk, de la new-wave et de la chanson libertaire. Ils partagent une passion, celle du rock dit alternatif, avec des figures tutélaires : « les Bérus » (Bérurier Noir) et « La Mano » (Mano Negra). Une idée précise de la fête, de la contestation, du concert avec ses rituels de pogo, de slam et d'interpellation du public. Pour la plupart d'entre eux, leur identité musicale et certains éléments de leur mode de vie tel que le cannabis, ainsi que le symbole du crocodile sont largement tatoués sur leurs corps. Une façon de dire très clairement ce que représente leur appartenance à Croco, un engagement social et personnel qu'ils dévoilent en public lorsque les corps se dénudent dans la chaleur et l'excitation de la scène.

7 Cette tanière, comme une peau de « croco " porte les traces des rencontres et de la vie, de l'histoire ordinaire. Son intimité et son intérieur affichent symboles et souvenirs. Ce local est organisé pour remplir diverses fonctions. Les instruments qui sont placés en demi- 
cercle autour du micro du chanteur font face au coin salon/bar constitué d'objets hétéroclites issus de la récupération, avec son frigidaire, son canapé, son fauteuil, ses tabourets, et le long du mur derrière le violoniste, un magnétophone à bande et une table de mixage ainsi que divers outils et cendriers qui sont posés sur une table d'écolier surplombée d'un énorme baffle de sono. On y trouve également des éléments qui participent au confort thermique (sous la tôle l'été l'ambiance peut y être suffocante) et acoustique telle que la laine de verre fixée aux murs et recouverte d'affiches et de divers petits objets usagés, le sol est lui recouvert de plusieurs couches de moquettes pour atténuer la réverbération, le froid et l'humidité.

\section{Un groupe très électroamplifié, la question du matériel}

Croco est un exemple assez ordinaire de groupe très électro-amplifié aux sonorités puissantes des battements répétitifs du batteur, et des sonorités électroniques amples, rugueuses, rappeuses et distordues, pas de place pour le silence, la musique est tendue, jouée d'un bout à l'autre avec la même intensité, la même énergie. L'instrumentarium se compose de cinq instruments électroamplifiés : deux guitares et une basse solid-body de marques et époques (références aux années 1950 et 1960) mythiques et américaines, une Gibson SG, une Gibson Lespaul et une Fender Precision, des micros dont celui très distinctif de la marque Shure du chanteur qui fait référence tout autant au rock'n'roll des années 1950 qu'au groupe mythique du punk anglais les Sex Pistols. Ce sont tous des outils traditionnels dans les groupes de rock célèbres et amateurs. Et trois instruments acoustiques : un violon amplifié avec une cellule, une paire de congas et la batterie qui sont amplifiées par des micros. La marque sonore des Crocos est d'abord celle des guitares et de la basse électriques et de la batterie associés à un chant à la voix «sale " éraillée psalmodiant des textes souvent contestataires en langue française. 
Les Crocos, un groupe aux sonorités puissantes de guitares et de batterie .

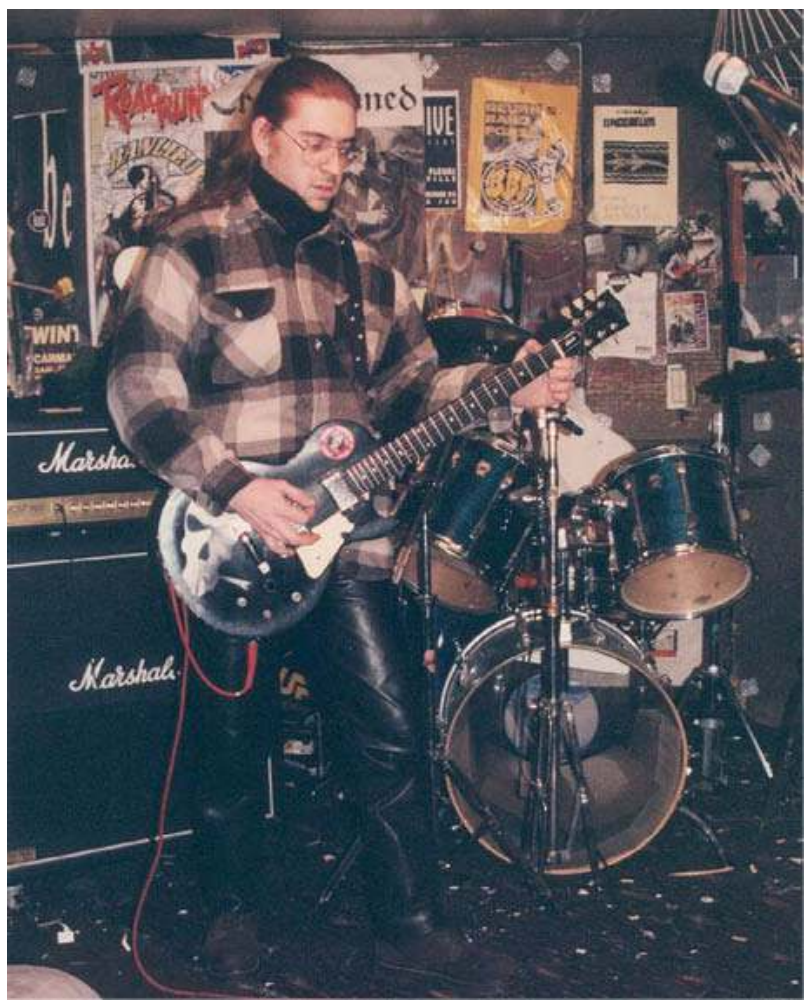

(C) Marc Touché, CNRS/Mupop

9 Ce lieu est daté, localisé et présente une mise en scène des amplis, percussions et musiciens. Il témoigne d'un ordonnancement de même que l'on dresse la table suivant les milieux sociaux, que l'on place les convives. Il montre les aspects fonctionnels tels que les procédés de rangement, de classement, d'affichage, de confort, de portage (caisse, valise, sacs plastiques, boîte à guitare) des instruments, des effets électroniques, des câbles, des outils. Il abrite un instrumentarium dont le choix correspond à des référentiels esthétiques, sonores, mais aussi à des logiques économiques. C'est un lieu clos, privatisé par un verrou précaire, relié à la société par le réseau EDF, l'électricité se répandant dans le local suivant des bricolages hors normes mais fonctionnant. Un local enserré entre :

10 - une forêt, symbole du calme qu'ils aiment et dont ils ont besoin pour récupérer de la fatigue musicale, tout à l'opposé du vertige sonore, de la fournaise de vibrations et décibels produits par les sonorités et battements qu'ils adorent jusqu'à l'épuisement ;

11 - les derniers logements des cités hlm, symbole de la présence des plus démunis, des malheureux et de la galère qu'ils chantent, crient et psalmodie dans leurs textes de chansons en concert et sur cassettes et $c d$. Galères que certains d'entre eux ont bien connues ou connaissent encore, tel le titre et le refrain « Compagnons de la misère »;

12 - une déchetterie, là où la ville se jette, s'oublie et où eux parfois vont chahuter, jongler, s'amuser pendant leur pauses musicales. Déchetterie dans laquelle ils peuvent s'approvisionner pour meubler leur local, y améliorer le confort. Un local fait de « récup » chez les copains, dans la famille, la décharge. La porte du local ouvre directement sur la décharge et des centaines de carcasses de pneus abandonnés en fin de vie. Alors que bien loin à Montluçon s'éteint progressivement l'un des grands sites industriel de mise au 
monde des pneumatiques Dunlop et se prépare lentement et par à-coups au grès des changements politiques le futur Mupop ;

Une semaine de chantier de recréation du local, à droite les affaires du sociologue, classeurs de photographies et boîtes contenant des objets de décoration.

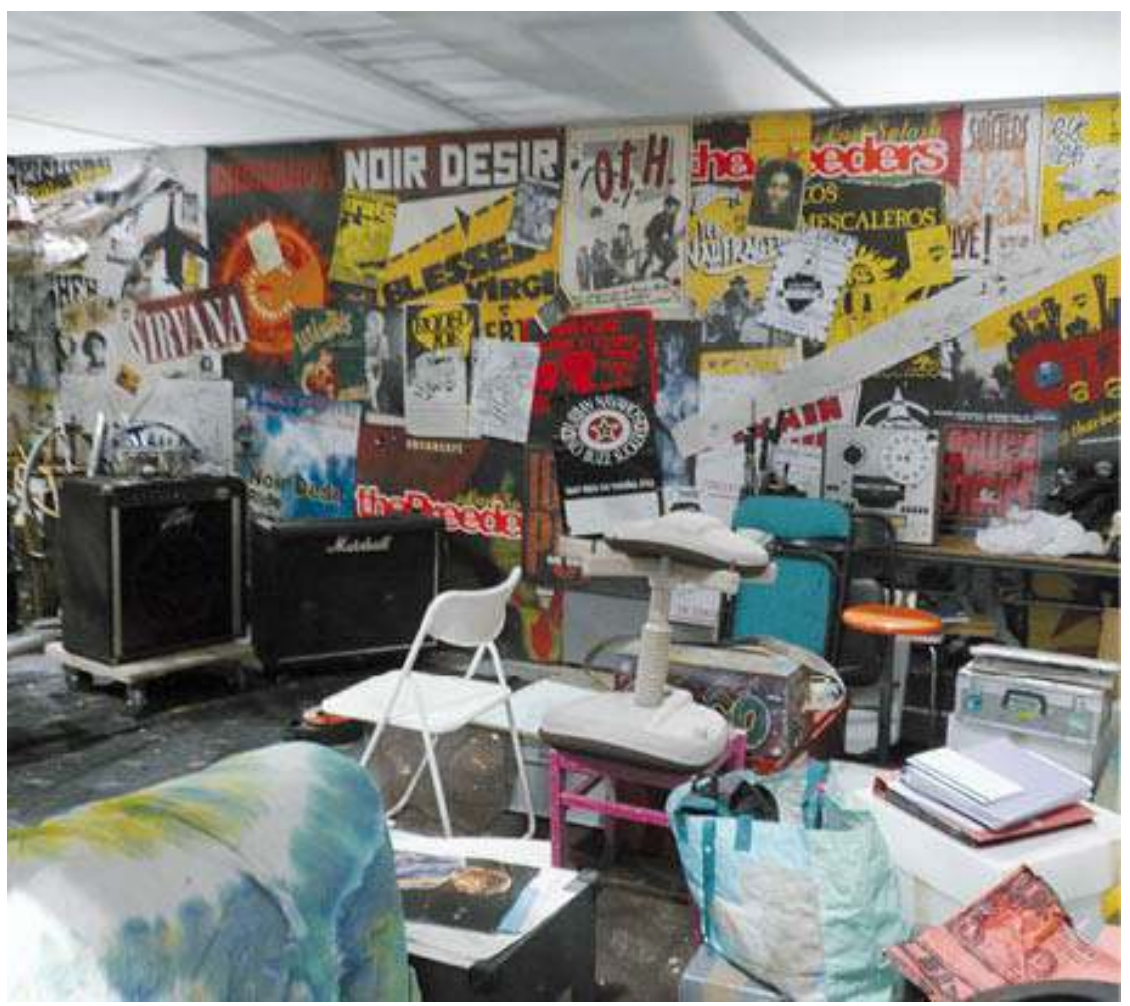

(c) Marc Touché, CNRS/Mupop

13 - un baraquement métallique du même type que le leur, distant de quelques mètres qui abrite une radio libre de la bande FM, symbole de l'ouverture sur le monde et de la diffusion des musiques produites artisanalement et industriellement. Le baraquement du groupe Croco est divisé en deux locaux de répétition qui communiquent par une porte intérieure située derrière le batteur. Ouverture que nous avions souhaité utiliser à l'occasion d'une présentation de ce local dans le Fnac de Lille en 2004 pour offrir au public un second point de vue sur le local. Cette ouverture a donc permis d'envisager de créer non artificiellement un sas vitré avec un point de vue original sur l'ensemble des instruments vus de dos et sur la décoration de trois murs, vision complémentaire du sas installé dans la porte d'entrée du local qui permet une vue panoramique sur l'instrumentarium et le coin salon-bar. Cette idée a ainsi été reprise pour la présentation définitive au Mupop, elle avait été testée positivement une seconde fois dans la présentation du local dans l'exposition Complément d'Objet en 2005 au château des Ducs de Bourbon (ancien musée des Musiques populaires de Montluçon).

14 C'est ainsi tout un programme d'anthropologie culturelle et urbaine qui est concentré dans ce petit local de quelques vingt mètres carrés qui a une façade très identitaire du côté intérieur de la déchetterie, graffé et tagué par les musiciens et leur public invités à des répétitions. Cette face du local est très colorée, elle informe sur le nom des habitants. Le nom du groupe s'y étale en lettres géantes, près de la porte telle une enseigne réservée aux initiés. La veille du déménagement, le groupe a joué une dernière répétition qui a été 
intégralement filmée en 16/9 et enregistrée en son numérique. Le démontage a été photographié. Des films, évoquant l'environnement du local, ses murs extérieurs et cette dernière répétition, sont diffusés dans des écrans plats intégrés aux murs du local qui sont recouverts de miroirs, dans sa présentation actuelle dans les parcours historiques et permanents au Mupop. Ces images sonorisées sont un complément nécessaire de la présentation du local puisqu'ils montrent à la fois: la place que se réserve chaque musicien, le mouvement des corps, la distance entre ceux-ci, les façons de porter l'instrument et de se situer vis-à-vis de son ampli et de ses haut-parleurs, les spécificités des sonorités et des rythmes du groupe. Le Mupop a veillé à avoir un parcours musical de qualité, avec par exemple de forts volumes sonores et des vibrations perçues par les pieds dans la salle traitant des festivals pop. Malheureusement, pour l'instant le mode de diffusion qui a été choisi par les scénographes pour sonoriser le local Croco, ne permet pas de se rapprocher de l'expérience réelle des sensations sonores, auditives et corporelles en immersion dans le local de répétition. La muséographie sonore et vibratoire reste un vaste champ de travail en ce qui concerne les formes d'expressions décibéliques et vibrationistes. Deux textes sociologiques accompagnent la présentation du local, ils sont collés sur les façades-miroir dans lesquelles se renvoient en abyme les vitrines environnantes, ainsi qu'un texte présentant la recherche in situ qui est placé à l'intérieur, en position basse derrière un sas vitré. Ces écrits tentent d'exprimer toute cette problématique aux publics qui sont en général très interpellés par cette « unité écologique » luxuriante et intrigante. Ils précisent également que ce local est un point de vue parmi d'autres sur la répétition. Qu'il faut se garder de tout amalgame.

Le local pendant sa recréation en 2013 par Marc Touché à partir de ses photographies d'enquête : au fond, l'un des deux sas vitrés pour le public.

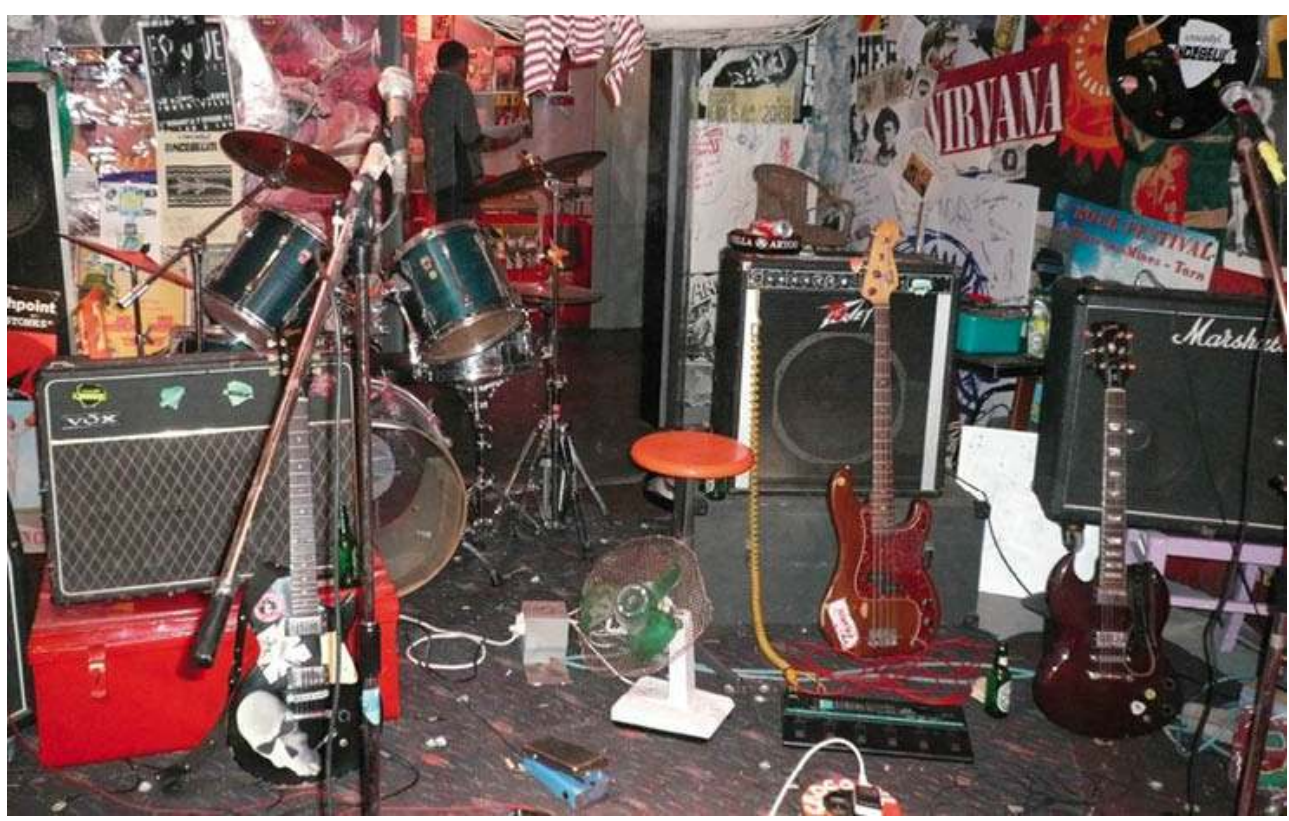

(C) Marc Touché, CNRS/Mupop

Dans sa vie avant l'entrée dans les collections du musée, ce local était déjà en lui-même un lieu de mémoire, de mise en scène de l'histoire d'un groupe, de ses proches et de ses réseaux musicaux. C'est sûrement aussi pour ces raisons que les musiciens de Croco ont trouvé cohérent d'accepter de jouer le jeu de cette entrée dans les collections du musée. 
La période de gestation de cette acceptation s'est étalée sur plusieurs mois. Petit à petit une amicalité et une solidarité intellectuelle sont nées entre le sociologue et les musiciens. L'ensemble du groupe a accompagné son entrée au musée, y compris par leur présence le jour du démontage (par l'équipe du Mupop et le sociologue), de l'emballage et du numérotage des pièces et de leur chargement dans un camion qui partait à la tombée $\mathrm{du}$ jour vers une nouvelle destination, emmenant le contenu du local et un bout de vie d'un groupe punk-rock vers une nouvelle aventure, une nouvelle histoire, une nouvelle identité : celle d'un ensemble d'objets de musée, qui va être administrativement étiqueté. Les cendriers, les moquettes ont été emballés avec tous les petits objets, restes, débris qu'ils contenaient. Aseptiser ces objets (en les débarrassant de ce qui est traditionnellement associé à l'image du désordre ou de la saleté) pour des raisons de conservation et de présentation mènerait à de terribles contresens sur ces sociabilités musicales. Croco est peut-être à l'échelle mondiale le premier groupe de musiciens vivant à voir son lieu - de réunion, de défoulement, d'entraînement, de travail, de création entrer dans une institution qui a pour vocation de conserver et rendre accessible et compréhensible aux générations actuelles et à venir un pan de la vie musicale des milieux populaires.

16 À la fin de la journée quand la coque métallique (qui ne faisait pas partie de l'acquisition puisque n'appartenant pas au groupe) se trouva mise à nu laissant apparaître sa précarité, sa rouille, ses trous en bas des parois; les yeux, la pâleur et les traits des visages des musiciens de Crocos avaient quelque chose de grave, nous étions tous mal. Ils venaient de vivre un choc différent des chocs électroamplifiés dont ils avaient l'habitude et la science, "on pensait pas que ça serait si fort, mais on est heureux et fiers de témoigner pour nos musiques...", de notre côté nous étions sous pression, avec un challenge énorme : emmener ce local transpirant l'humain dans une autre vie au milieu d'objets de musée dans des réserves silencieuses, dans des salles ouvertes à la curiosité ou à l'indifférence des publics, objets inanimés et souvent décontextualisés et aseptisés que l'on contemple derrière les vitrines bien propres. Sur la route vers Montluçon nous avions comme une bombe entre les mains : le chaud à la rencontre du froid, le sale à celle du propre, le sans légitimité culturelle institutionnelle et la future " unité écologique » inventoriée et ayant acquis une légitimité culturelle inaliénable.

Présenté dans les parcours historique et permanent au Mupop ce local continue d'avoir une âme, en cela personne parmi les visiteurs ne s'y est trompé (des gens demandant, « ils viennent de jouer?» ou, «ils vont jouer à quelle heure?»); ce n'est pas une reproduction, une fiction, mais il s'agit de la restitution dans un musée d'une « unité écologique » livrant ses secrets : son fouillis au sol, ses iconographies entremêlées sur les murs, sa luxuriance de couleur, sa décoration, son mobilier « ici c'est un peu le bordel, on fait le grand ménage quelques fois dans l'année... ». Dans cette nouvelle vie au cœur d'une salle du Mupop, cet espace est comme resté animé de vie sociale, quelque chose d'étonnant : ce lieu transpire l'humain près de vingt ans après son démontage. En tant que sociologue découvreur du local, j'ai eu l'honneur et le bonheur de le recréer, de lui offrir une nouvelle vie à partir des photographies de mes enquêtes de terrain. 


\section{Le patrimoine immatériel, celui de la musique jouée «tout d'oreille»}

Dans ce local nous ne trouvons pas de partition, seules des listes (playlist) de morceaux composant le répertoire de concert se trouvent sur les murs en souvenir ou au sol si elles sont d'actualité. En situation de répétition et de concert les musiciens de Croco travaillent " tout d'oreille ", le local abrite des tenants de la tradition orale, on apprend par cœur, on tchatche, on se réfère à des souvenirs communs des avancées des dernières répétitions, souvenirs qui sont l'objet de nombreux accrochages, chacun en ayant sa version. Ces morceaux sont presque tous créés, construits, affinés dans le cadre d'improvisations collectives. L'incertitude est un élément important dans ces musiques jamais fixées par l'écrit.

\section{Limites sensorielles dans la présentation du local}

Le local Croco a une odeur particulière, qui est propre aux lieux de musiques amplifiées ne possédant pas d'aération: un mélange sucré salé, âcre et doux à la fois, une odeur d'humidité, de renfermé, une composition complexe qu'il faudrait bien restituer un jour aux visiteurs pour qu'ils découvrent les caractéristiques sensuelles d'un tel lieu bricolé. Ce sont à la fois les restes d'odeurs corporelles (jouer énergiquement est comme un sport), de senteurs d'électroniques des quatre amplificateurs à lampes et transistors, des quatorze haut-parleurs, d'ébénisterie des huit enceintes, des cendriers débordants de mégots, des restes de cigarettes et de joints jonchent également le sol, comme des traces rassurantes de la vie passée, de fonds de bières dans lesquels peuvent macérer des mégots, des papiers et tissus humides et poussiéreux, des encres des affiches, de vieilles moquettes, de laine de verre. Une fois le local a failli être nettoyé de ce qui fait partie de son charme de son identité, lors d'un montage d'exposition, un matin nous avions arrêté à temps la femme de ménage qui voulait aspirer les moquettes, les mégots, les restes de cordes de guitare, les bouts de médiators, les capsules de bières... Je réfléchi à proposer un odoramat qui offre aux publics un autre aspect de la densité sensorielle de ces univers de sociabilités électroamplifiées. Il s'agit ici d'un type d'ambiance souhaitée, propice à la création collective dans un contexte social particulier. L'endroit est toujours enfumé, l'univers sonore, visuel et olfactif y est saturé de sensations fortes. 


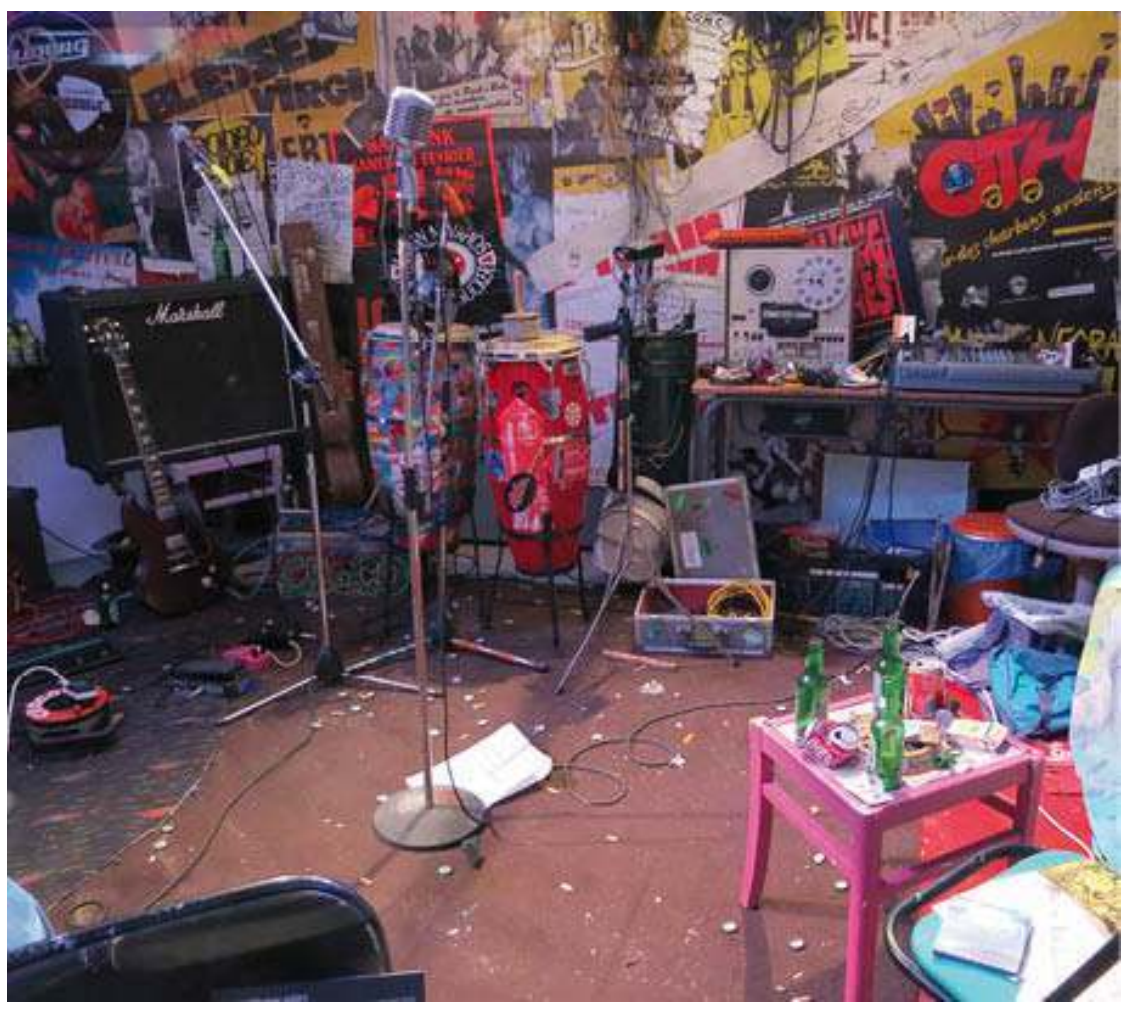

(c) $\mathrm{OCIM} / \mathrm{SL}$

\section{Créer des collections contrastées, éviter autant que faire se peut la folklorisation de sujets qui s'y prêtent particulièrement bien}

Il y a aujourd'hui urgence et nécessité de faire une socio-histoire des «musiques amplifiées » : des générations d'acteurs disparaissent de plus en plus régulièrement. Une socio-histoire vue de France, et non une histoire du rock américain ou anglais qui nous est servie comme si elle était notre histoire, ce qu'il faut réaliser c'est une histoire de ces musiques dans leurs usages ici, hier et maintenant. Il nous faut explorer comment des cultures musicales venues d'ailleurs ont rencontré celles d'ici, quelles sont les particularités de ces phénomènes d'appropriation, d'adaptation, quels en sont les acteurs, dans quels espaces et dans quelles dynamiques. Nous avons aujourd'hui le recul nécessaire pour faire des biographies qui permettent d'étudier des processus, des carrières, des cheminements. Ce travail en collaboration avec les musées est un vaste chantier de fouilles sur le contemporain et la vie ordinaire. Ces recherches sont l'occasion de collecte in situ et documentées.

Ces travaux sont au cœur de l'une des grandes difficultés pour les musées lorsqu'ils créent des expositions. Il s'agit d'appréhender la matière sonore, ici avec une dimension sociologique particulière: pas celle légitimée par des siècles d'us et coutumes, pas celle légitimée par les institutions socialisatrices traditionnelles, mais le sonore sauvage, indiscipliné, celui qui est en pleine invention que l'on ne sait encore comment cataloguer, 
celui qui crie, qui sature et signifie la contestation des ordres établis, celui porté entre autre par la jeunesse (génération après génération) et parfois par ceux qui souffrent et ne trouvent plus dans les mots la possibilité de communiquer. Sonore du blues, du punk, du rap qui est produit à l'aide d'instruments, de «machines » électro-amplifiés. Sonore utilisant souvent comme jamais dans l'histoire humaine les extrêmes graves dit « subbass "; sonore électro-amplifié permettant les usages les plus paroxystiques, terrain d'aventures des raves, des sous-sols, de la techno, du punk, du rap et du heavy métal, du trash...il a pénétré les espaces domestiques, public, il est porté au corps et décliné en festivals gigantesques et cultures de caves, garages et studio high-tech. Le Mupop est face à un immense challenge: être le témoin, créer des collections et être le lieu de questionnement concernant l'une des dernières grandes transformations des modes de vie (l'électroamplification) avant celle d'Internet.

Le travail sur la mémoire, réalisé avec les musées à partir de recherche-collecte, peut-être l'une des voies d'accès à la constitution d'une connaissance de pratiques rarement étudiées, dont on peut penser que jusqu'à il y a peu encore, elles étaient considérées comme insignifiantes, dérisoires, anecdotiques. Ce travail avec les musées contribue en quelque sorte à accorder une légitimité nouvelle à cette grande aventure humaine du XXe siècle qui ne peut être réduite uniquement à ses dimensions spectaculaires du vedettariat qui sont en général célébrées dans les expositions de musique pour attirer du public. L'expérience montre que le local qui lui n'est pas attractif en matière de communications grand public est par contre l'un des temps très forts de la visite des publics. Un temps d'étonnement, quelque chose d'extraordinaire qui résonne en chacun, qui interroge, sur l'ordre, le désordre, la complexité, les ambiances, la création, les sociabilités, une installation qui fait discuter les gens entre eux, les enfants avec les parents. Un univers si différent des représentations dominantes des ensembles musicaux tels que les "fanfares", les petits "orchestres» de musique classique, Croco est un "groupe » constitué d'abord sur les bases de l'amitié.

\section{Mupop : le musée des Musiques populaires}

Le Mupop a ouvert ses portes au public le 21 juin 2013. Seul musée en France consacré aux musiques populaires, il en décline l'histoire sur $3300 \mathrm{~m} 2$, de la fin du XVIIIe siècle à nos jours. 


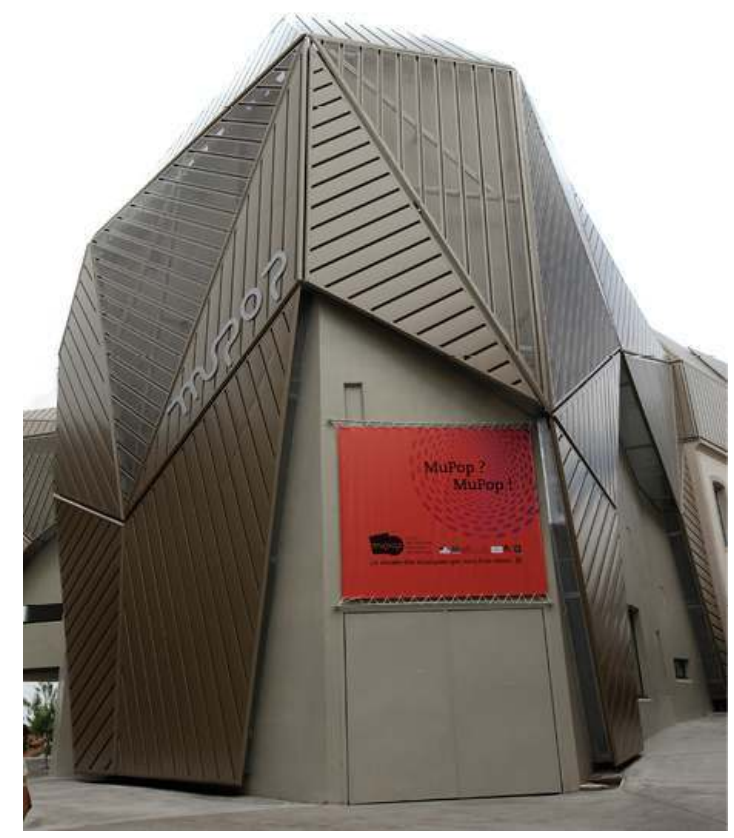

(c) Ville de Montluçon

Le Mupop se compose de deux principaux parcours. Un parcours musical consacré aux répertoires musicaux et à leur lien avec la société qui fonde leur popularité. Ce parcours met en scène la musique autour de ses lieux et usages. On y rencontre ainsi un parquet de bal, une chambre d'adolescent des années 1960, un local de répétition d'un groupe punk... tandis que des spectacles musicaux se déclenchent dans chaque espace toutes les 7 minutes, restituant ces musiques au temps de leur popularité. Ce parcours met également l'accent sur la problématique de la diffusion de la musique par les technologies du XXe siècle : le disque, la radio, la télévision et enfin l'ordinateur.

Le second parcours, plus classique, est consacré aux instruments de musique eux-mêmes autour des 3 collections fondamentales du Mupop: les vielles, les cornemuses et les guitares électriques. Ce parcours s'accompagne de nombreux montages vidéos, modèles de démonstration et ateliers de lutherie... 
La reconstitution d'un atelier de luthier

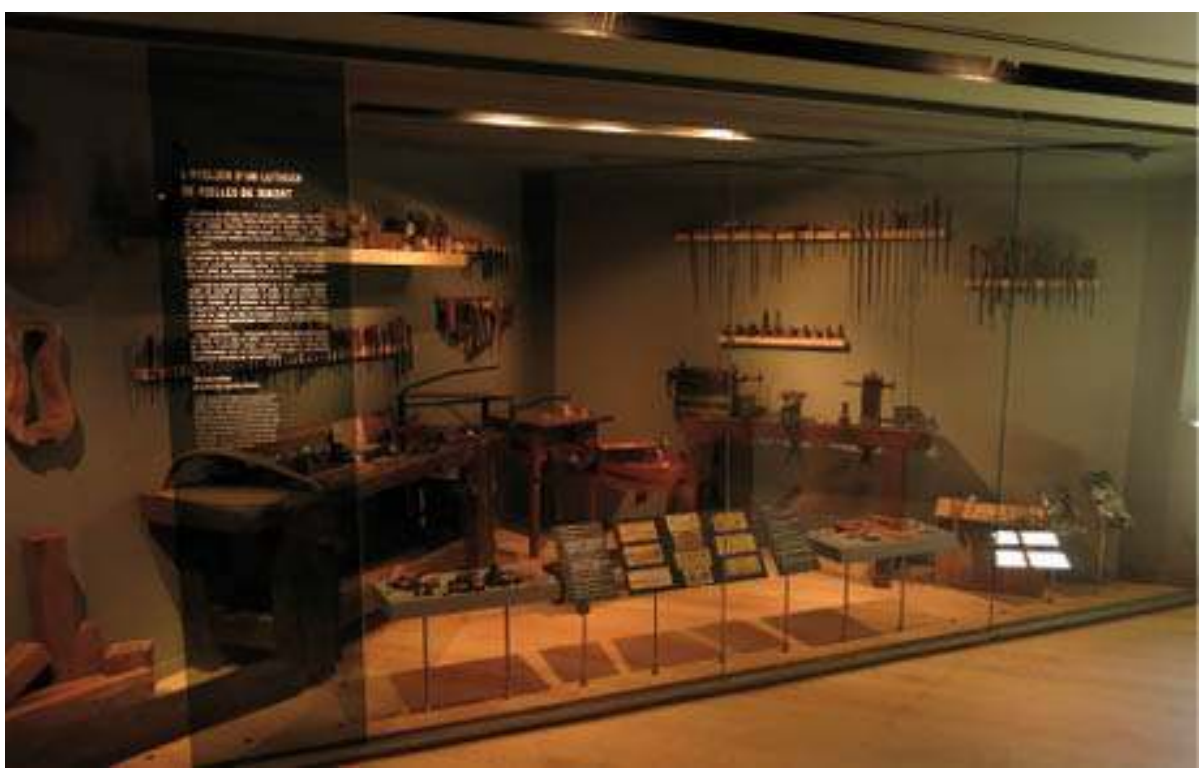

(c) $\mathrm{OCIM} / \mathrm{SL}$

26 Ces deux parcours sont complétés par un espace expérimental dédié aux technologies numériques et par un vaste espace d'expositions temporaires se renouvelant chaque année et qui fut en 2014 consacré au « Disco ».

27 Musée dédié à la musique, le Mupop se devait de la faire écouter dans les meilleures conditions, ainsi chaque visiteur reçoit-il à son arrivée un casque raccordé à un petit boîtier qui lui permet ainsi d'accéder à la musique dans l'ensemble du musée. En pointant les objets il en écoute donc le contenu sonore à sa guise, composant son propre programme musical. Huit heures de musique sont ainsi disponibles.

28 Le Mupop est donc un musée qui se vit et s'expérimente par soi-même, et outre la nature de son contenu, son mode de fonctionnement contribue grandement à son succès auprès du public. 
Une partie de la collection de guitares électriques amplifiées.

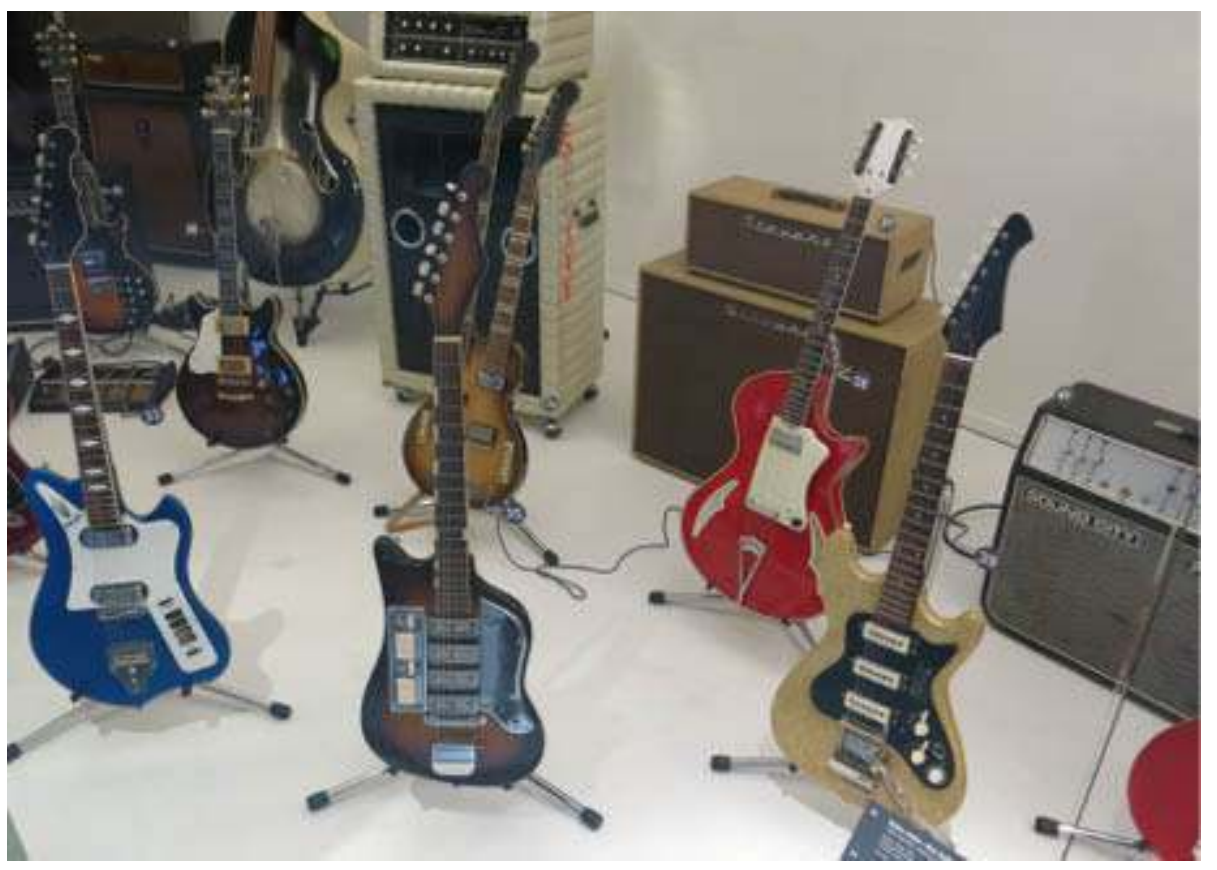

(c) $\mathrm{OCIM} / \mathrm{SL}$ conférences et projections. Il reçoit aussi un grand nombre de publics scolaires pour lesquels un dispositif de médiation se met peu à peu en place en collaboration avec les enseignants.

\section{NOTES}

1. Référence aux parcours permanents du MNATP dans lequel j'ai travaillé de 1995 à 2006.

2. Touché, M. Ethnosociologie des musiciens utilisant des instruments électroamplifiés, in Guitares, guitaristes et bassistes électriques, catalogue d'exposition, musée des Musiques populaires, Montluçon, 1998, pp. 39-69.

3. Touché, M. Connaissance de l'environnement sonore urbain, l'exemple des lieux de répétition. Rapport de recherche pour le ministère de l'Environnement. Travaux de recherche, $\mathrm{n}^{\circ} 8, \mathrm{CRIV} /$ CNRS, 1994.

4. William Saadé était conservateur.

5. Touché, M. Musique et vie quotidienne, Annales de Vaucresson, $n^{\circ} 28$, Adolescence et socialisation, 1998/1, pp. 123-158.

6. Touché, M. Mémoire Vive 1. CEF-MNATP-CNRS, Association Musiques Amplifiées Annecy, 1998. 
7. Moreau, N. et Touché, M. Rockin'Laval 1960-2000. Catalogue de l'exposition 2009-2010, avec dvd, ADDM53, Mupop, CNRS.

8. Ces dimensions sont développées dans : Touché, M. Les musiques amplifiées s'exposent et s'invitent dans les musées, Question de communication, n²2, 2012, pp. 57-86. Touché, M. Muséographier les musiques éléctroamplifiées. Pour une socio-histoire du sonore, Réseaux, vol. 25, 2007, pp. 97-142.

9. Lory, M.-C., Grenet, S., Sabatier, M. et Touché, M. Guitares Jacobacci, un atelier de lutherie à Paris 1924-1994. Paris : Somogy et Montluçon : Mupop, 2006.

\section{RÉSUMÉS}

Comment rendre compte des pratiques musicales amateurs dans l'espace muséal? Comment muséographier les musiques électroamplifiées?

À partir d'une recherche-collecte et d'une enquête sociologique menée avant et pendant le transfert au musée des Musiques populaires de Montluçon d'un local de « répète » d'un groupe de musique, l'auteur tente de répondre à ces interrogations.

\section{INDEX}

Mots-clés : musique, musée

\section{AUTEUR}

\section{MARC TOUCHÉ}

sociologue, chercheur associé IDHES CNRS, chargé de mission au musée des Musiques populaires (Mupop) de Montluçon

marctouche@orange.fr 